\title{
Die literarische Konstruktion von Gedächtnis und Erinnerung in Elias Canettis Autobiografie
}

\author{
Dr. Shaimaa Ahmed Elsaghir Tawfik \\ Dozentin für die deutsche Literatur \\ Abteilung für Germanistik \\ Al-Alsun Fakultät, Sohag Universität
}

\begin{abstract}
Deutsch):
Das Thema Gedächtnis ist im Rahmen der Literatur- und Kulturwissenschaft von großer Bedeutung. Daher ist die Erinnerungspraxis und deren Praxis $\mathrm{zu}$ einem interdisziplinären, internationalen und vor allem gesamtkulturellen Phänomen geworden. Die vorliegende Untersuchung setzt sich mit der literarischen Konstruktion von Gedächtnis und Erinnerung anhand der autobiografischen Schriften Elias Canettis auseinander. Dabei zielt die Studie darauf ab, die Rolle des Gedächtnisses in Canettis autobiografischen Schriften $\mathrm{zu}$ eruieren. Die Gedächtnistheorien der Kulturwissenschaft, wie sie von Ansgar Nünning, Jan und Aleida Assmann und Astrid Erll entwickelt wurden, formulieren den theoretischen Rahmen dieser Studie. Canettis Die gerettete Zunge scheint mir als Beispiel zur Besprechung des angeführten Themas geeignet zu sein, weil der Autor darin das Gedächtnis als Werkzeug des Schreibens gut dargestellt. Zudem gilt die Autobiografie von Canetti als wichtiges Beispiel für die literarische Konstruktion von Gedächtnis und Erinnerung.

Schlüsselwörter: Gedächtnis, Erinnerung, Autobiografie, Elias Canetti
\end{abstract}

\begin{abstract}
Englisch)
The topic of memory is of great importance within the framework of literary and cultural studies. Therefore, the practice of memory and its practice has become an interdisciplinary, international and, above all, pan-cultural phenomenon. The present study deals with the literary construction of memory and recollection on the basis of Elias Canetti's autobiographical writings. In doing so, the study aims to explore the role of memory in Canetti's autobiographical writings. The theories of memory in cultural studies, as developed by Ansgar Nünning, Jan and Aleida Assmann and Astrid Erll, formulate the theoretical framework of
\end{abstract}


(ISSN : 2536 - 9555)

this study. Canetti's The tongue set free seems to me to be suitable as an example for discussing the topic cited, because in it the author depicts memory well as a tool of writing. Moreover, Canetti's autobiography is considered an important example of the literary construction of memory and recollection.

Key Words: memory, recollection, autobiographical writings, Elias Canetti

$$
\begin{aligned}
& \text { البناء الأدبي للذاكرة والتذكر في السيرة الذاتية للكاتب إلياس كانيتي } \\
& \text { د. شيماء أحمد الصغير توفيق } \\
& \text { مدرس الأدب الألماني ـ قسم اللغة الألمانيةــ كلية الألسن - جامعة سوفيق لهاج }
\end{aligned}
$$

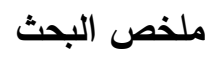

يحاول هذا البحث دراسة موضوع البناء الأدبي للذاكرة والتذكر ، حيث إن دراسة موضوع

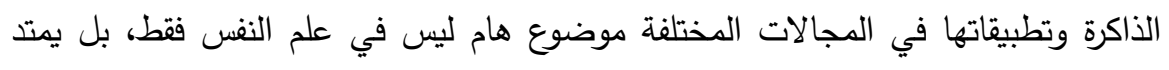
دراسته وتتاوله في مجالات الدراسات الثقافية و الأدبية، ويركز هذا البحث علي دورئ دراسة موضوع البناء الأدبي للذاكرة والتذكر من خلال السيرة الذاتية للكاتب إلياس كانيتي والحائز

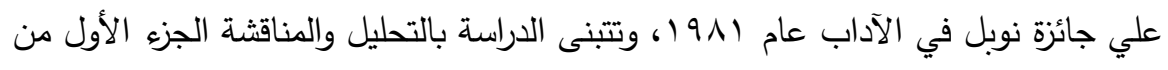

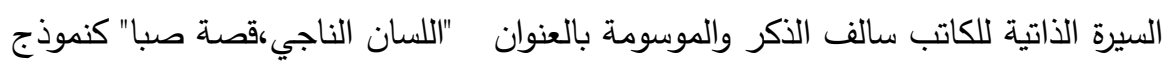
تطبيقي"، وقد تم تقسيم الدراسة إلى أربعة فصول، يتناول الفصل الأول الذاكرة وأنواعها والفرق بينها وبين التذكر، ويشكل ذلك الإطار النظري للدراسة الذي يستتد لبعض الدض الدراسات

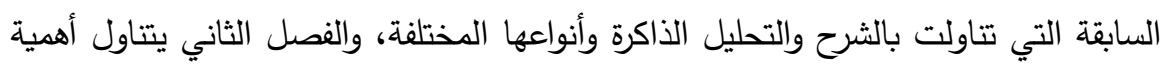
التذكر في الأدب والسيرة الذاتية، أما الفصل الثالث فيتناول الذاكرة والتذكر من خلال أعمال الأديب العالمي إلياس كانيتي، والفصل الرابع و الأخير ينقسم إلى مبحثين حيث يقدم

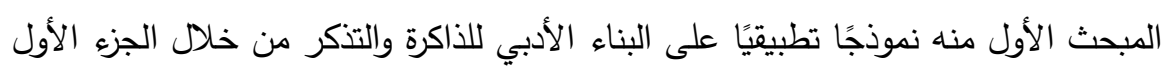

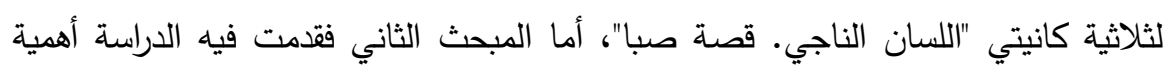
التذكر بالنسبه للراوي وناقثت دور الذاكرة والتذكر في بناء الإطار الزمني والمكاني لرواية

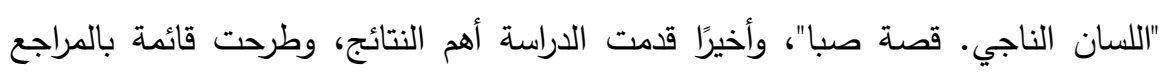

$$
\text { والمصادر التي استعانت بها؛ لتتديم العمل حتى نهايته. }
$$

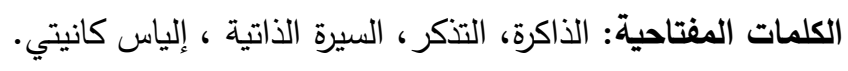


Die literarische Konstruktion von Gedächtnis und Erinnerung in Elias

Canettis Autobiografie

Dr. Shaimaa Ahmed Elsaghir Tawfik

مجلة وادي النيل للاراسات والبحوث الإنسانية والاجتماعية والتريوية (مجلة علمية محكمة)

\section{Die literarische Konstruktion von Gedächtnis und Erinnerung in Elias Canettis Autobiografie}

\section{Einführung}

Die Autobiografie ist eine der prosaischsten Formen des Gedächtnisgebrauchs im literarischen Schreiben. Darüber hinaus gibt es Geschichten, Romane oder Gedichte, deren Inhalt und Aufbau von dem abhängt, was das menschliche Gedächtnis zu einem bestimmten Thema, Ereignis oder bestimmten Personen bewahrt hat. Es war immer ein Teil von Elias Canettis Schreibmethode, etwas von seinen eigenen Erfahrungen in seine Arbeit zu übertragen, doch Die gerettete Zunge wird unweigerlich zur Autobiografie, weil es sein Leben und seine Philosophie in ein geschlossenes Ganzes einbindet. Stil und Substanz sind ebenso aussagekräftige Indikatoren für die Präsenz des Autors im Werk. Canettis Beziehung zur Arbeit als Autobiograf wird noch selbsterklärender, weil er sich in der Doppelrolle von Kind und Erwachsener kreativ in den Text einbringt. Szenen aus der Kindheit werden durch das Prisma des Erwachsenen und die ausgereifte Perspektive des Autors verstärkt, geklärt und kreativ differenziert. Canetti beschäftigte sich nicht nur mit der Erinnerung an Dinge in der Vergangenheit, sondern auch mit der Anwendung und Diskussion seiner modernen Ideen über Philosophie, Psychologie und Ästhetik. Seine Synthese von Erfahrungen ergibt ein Meisterwerk von Tiefe und Dimension, in dem Canetti sich selbst, Vergangenheit, Gegenwart und Zukunft umschreibt.

Elias Canettis Die gerettete Zunge (1977) demonstriert Behauptungen über die Fiktionalität der Autobiografie, nämlich dass die Produktion einer autobiografischen Erzählung zwangsläufig ein Prozess der Auswahl und Interpretation statt der Darstellung eines Lebens, ,wie es ist“" darstellt. Dieser Beitrag soll unser konventionelles Verständnis von Autobiografie und ihrem 
(ISSN : 2536 - 9555)

Verhältnis zu Fiktion, Gedächtnis und Erinnerung untersuchen. Canettis Verständnis der zusammengesetzten Struktur des Gedächtnisses informiert meine Analyse über seine Fixierung auf die Bücher und Erzählungen, denen er als Kind begegnet ist und wie diese nicht nur mit seinen Kindheitserinnerungen verflochten sind, sondern auch einen Rahmen bieten, mit dem er die Erzählung seines Lebens ordnet.

\section{Gedächtnis und Erinnerung}

Welche Stellung nehmen Gedächtnis und Erinnerung im Rahmen des autobiografischen Schreibens ein? Was verstehen wir unter Begriffen wie Gedächtnis und Erinnerung? Um diese Fragen zu beantworten, erscheint es sinnvoll, zuerst den Begriff Gedächtnis und seine Konzepte zu erklären. Gedächtnis und Erinnerung besetzen einen bedeutenden Stellenwert im Rahmen der kulturwissenschaftlichen Forschung (vgl. Erll, S. 7).

Das Gedächtnisforschung lässt sich primär der Psychologie, insbesondere der kognitiven Psychologie zuordnen. Daher ist es sehr wichtig für die Analyse des menschlichen Verhaltens, da es mit den vergangenen beibehaltenen Erfahrungen, Gefühlen, und Gedanken verbunden ist, die explizit oder implizit das unmittelbare und zukünftige Leben des Individuums beeinflussen, d. h. das Gedächtnis ist mit der Geschichte und der Kultur sowohl individuell als auch kollektiv verbunden. Auf einen einheitlichen Begriff scheint „Gedächtnis“ nicht zu bringen zu sein. Man kann es definieren ,als die Fähigkeit, Erlebtes und Gelerntes zu behalten, aber auch zu vergessen, um Neues aufnehmen zu können; Erinnerung ist demgegenüber der Akt, sich im Gedächtnis Gespeichertes bewusst zu machen“ (Assmann: Gedächtnis/Erinnerung, S. 233). Gedächtnis ließe sich als „Prozesse und Systeme, die für die Einspeicherung, die Aufbewahrung, den Abruf und Anwendung von Informationen“ begreifen (Gruber 2018, S. 10). Hierbei können drei Haupttypen vom Gedächtnis unterschieden werden, die für unterschiedliche Dimensionen unserer Erinnerung verantwortlich sind: Das 
Die literarische Konstruktion von Gedächtnis und Erinnerung in Elias

Canettis Autobiografie

Dr. Shaimaa Ahmed Elsaghir Tawfik

مجلة وادي النيل للاراسات والبحوث الإنسانية والاجتماعية والتربوية (مجلة علمية محكمة)

sensorische Gedächtnis (Ultrakurzzeitgedächtnis) bedeutet die Fähigkeit der fünf Sinne, Reize (Wörter, Bilder, Geräusche etc.) zu empfangen und sie in Informationen umzuwandeln, um sie dann in das Kurzzeitgedächtnis zu übertragen. Diese Informationen werden nachträglich aufgerufen, wenn das Gehirn den gleichen oder ähnlichen Reizen ausgesetzt ist. Das Kurzzeitgedächtnis (KZG) repräsentiert die zweite Phase, in dem die aus dem sensorischen Gedächtnis angekommenen Informationen gespeichert werden. Es hat eine begrenzte Kapazität sowie eine kurze Dauer der Aufbewahrung von Informationen. Dabei dient es zur Übertragung der Informationen ins Langzeitgedächtnis. Unter dem Langzeitgedächtnis wird das allgemeine Konzept des Gedächtnisses verstanden. Es gilt als die komplizierte und die vielfältige Art des Gedächtnisses, das große Mengen an Information in sich aufnehmen und auch für eine lange Zeit über das gesamte menschliche Leben behalten kann.

Nach dem Inhalt kann man das Gedächtnis in vier unterschiedliche Arten untergliedern: das semantische Gedächtnis, das mit Konzepten, Wahrnehmungen, Ideen und dem erlernten Wissen verbunden ist und einen starken Bezug auf die Umwelt des Individuums hat (vgl. Tulving 1979, S. 9). Die zweite Art ist das episodische Gedächtnis, das mit einzigartigen, greifbaren Erfahrungen aus der Vergangenheit verbunden ist, während sich das semantische Gedächtnis auf das Wissen des abstrakten Menschen über die Welt um ihn herum bezieht, sodass dieses Wissen nicht mit einer bestimmten Zeit zusammenhängt. Dementsprechend bezieht sich das semantische Gedächtnis auf das allgemeine Wissen des Individuums über verschiedene Ereignisse und Informationen auf der ganzen Welt, d. h. es dient als allgemeine Informationsbasis, die man mit anderen teilt, die bekannt, für alle zugänglich und überprüfbar ist, während das episodische Gedächtnis aus den eigenen Erfahrungen des Individuums besteht und sich an die Vergangenheit erinnert, mit einem spezifischen Fokus auf Zeit und Raum (vgl. ebd.) Das 
(ISSN : 2536 - 9555)

prozedurale Gedächtnis ist hingegen für die Speicherung von Bewegungsabläufen verantwortlich, wie z. B. Fahrradfahren, Autofahren, Schuhe zubinden und Schwimmen. Dabei handelt es sich um einen Speicher für Verhaltensroutinen und automatisierte Fertigkeiten (vgl. Gruber 2018, S. 69). Zuletzt haben wir das assoziative Gedächtnis, unter dem die Fähigkeit verstanden werden kann, die Beziehung zwischen nicht zusammenhängenden Dingen wie dem Namen einer Person, die wir gerade getroffen haben, oder dem Aroma eines bestimmten Parfums zu lernen und sich daran zu erinnern (vgl. Suzuki 2005).

Es ist üblich, Gedächtnis mit Erinnerung zu verwechseln. Gedächtnis ist die Speicherung und Filterung von wahrgenommenen und sensorischen Informationen aus der äußeren Umwelt durch den Prozess der Aufmerksamkeit und die Speicherung dieser Informationen, wenn dies nötig ist, während Erinnerung das Erinnern und der Abruf dessen, was im Langzeitgedächtnis gespeichert wurde, und der Grund für diese Exposition, die zur Aufregung und zum Abrufen dieser Ereignisse führte. Das Erinnern kann dementsprechend als ein prozesshafter Vorgang betrachtet werden (vgl. Erll 2017, S. 14).

\section{Die literarische Erinnerung und Autobiografien}

Jeder von uns hat eine Biografie, eine Geschichte, die sich von Zeit $\mathrm{zu}$ Zeit an ihre Ereignisse erinnert, eine Sammlung von Erinnerungen an ihre Situationen, Themen und Menschen, Träume, Bestrebungen, Wahrnehmungen und Ideen, von denen viele erinnert und viele vergessen werden. Das Erinnern ist daher eine Besonderheit der Menschen, da es einer der höchsten mentalen Prozesse ist, die eine einflussreiche Rolle im menschlichen Leben spielen. Wenn wir ein wenig weiter schauen, werden wir feststellen, dass jeder Aspekt unseres Lebens auf Erinnerung basiert, darunter das Erlernen von Wissen, Wissenschaft und Fähigkeiten sowie von Bräuchen, Traditionen und Beziehungsbildung, aber noch wichtiger ist, dass auch Anbetung und religiöse Rituale auf Bewahrung und Erinnerung basieren. 


\section{Die literarische Konstruktion von Gedächtnis und Erinnerung in Elias \\ Canettis Autobiografie}

Dr. Shaimaa Ahmed Elsaghir Tawfik

مجلة وادي النيل للاراسات والبحوث الإنسانية والاجتماعية والتريوية (مجلة عمية محكمة)

Deshalb stand das menschliche Gedächtnis im Vordergrund der Aufmerksamkeit von Wissenschaftlern und Forschern.

Das Gedächtnis ist eines der wichtigsten Werkzeuge des Schreibens, ohne das wir nicht über einen literarischen Text sprechen können, insbesondere über einen narrativen Text, der nicht ohne Erinnerung verifiziert werden kann, obwohl das Schreiben nicht so sehr eine treue Erinnerung ist, sondern mit einer wahren Erinnerung beginnen kann, aber letztendlich seine eigenen und rebellischen Welten schafft. Trotzdem sind die literarische Erinnerung und Autobiografien laut Klaus Schenk nicht so eng miteinander verbunden. Er ist der Auffassung, dass die literarische Erinnerung eher für andere literarische Gattungen wie z. B. Tagebücher, Memoiren und andere Formen zutrifft und die modernen Autobiografien vielmehr mit fiktionalen Schreibweisen und imaginärer Dimension verbunden sind (vgl. Schenk 2009, S. 19).

\section{Gedächtnis und Erinnerung im Schreiben Elias Canettis}

Wie bereits erwähnt, wurde für diesen Beitrag der ersten Band der dreiteiligen Autobiografie Die gerettete Zunge. Geschichte einer Jugend des bulgarisch-österreichischen Schriftstellers Elias Canetti, eines der berühmtesten Schriftsteller der deutschsprachigen Literatur, ausgewählt. Er erhielt 1981 den Nobelpreis für Literatur. Sein literarisches Schaffen umfasst einen Roman, drei Dramen, Essays, Reiseberichte, Tagebücher und einen dreiteiligen Autobiografie-Zyklus. Zudem interessiert er sich für Philosophie, Psychologie und Soziologie. Während seines Lebens bewegte er sich zwischen mehreren europäischen Städten, darunter Manchester, Wien, London, Paris, Frankfurt und Zürich, was seine Schriften positiv beeinflusste, die eine tiefe und umfassende Vision der menschlichen Realität im Allgemeinen und der europäischen Auswirkungen im Besonderen zum Ausdruck brachten. Canetti kritisierte Opportunismus, Klassismus, Krieg und Tyrannei, 
(ISSN : 2536 - 9555)

verteidigte die Identität des europäischen Menschen und die europäische Kultur und war fasziniert von britischen Ideen, deshalb führte er mit seinem philosophischen Buch Masse und Macht (1960) satirisch eine Analyse der damaligen gesellschaftlichen Umstände ein.

Canetti hat dazu tendiert, nicht nur in seinem AutobiografieZyklus, sondern in allen seinen Texten auf gleichzeitig distanzierte und intime Weise autobiografisch zu schreiben (vgl. Barnouw 1979, S. 1). Gedächtnis und Erinnerung werden in Canettis Werk explizit und manchmal implizit gebraucht.

Elias Canetti ist als Autobiograf und Schriftsteller besonders geprägt von seinen Welterfahrungen, insbesondere von Ereignissen, Orten, Menschen, Gesprächen und Lektüre, somit gelang es ihm im Hinblick auf seine autobiografische Erinnerung $\mathrm{zu}$ dichten und Geschehen aus seinem Gedächtnis zum autobiografischen Subjekt seiner frühen Werke zu machen (vgl. Di Maio 2004, S. 175). Wenn wir seine Leistung im Literaturbetrieb betrachten, erkennen wir, dass er über Gegenstände aus seinem Gedächtnis schreibt und in fast allen seinen Texten einen autodiegetischen Erzähltypus verwendet, der ebenfalls Anspruch auf Authentizität, Subjektivität und Ambivalenzen zeigt. Kritiker haben oft darauf hingewiesen, dass der Roman Die Blendung, das Drama Hochzeit und vor allem sein philosophisches Buch Masse und Macht autobiografisches Erinnern und sein Ich immer wieder neu darstellen, um es an die veränderten Lebensumstände anzupassen.

Die dreibändigen Erinnerungen, eine der besten Autobiografien des Jahrhunderts, zeugen von der großen Kraft, mit der Canetti für das Leben und gegen den Tod kämpft. Im zweiten Teil seiner Autobiografie Die Fackel im Ohr formuliert er positiv, welche Bedeutung das Erinnern überhaupt im Leben des einzelnen Menschen hat: 
Die literarische Konstruktion von Gedächtnis und Erinnerung in Elias

Canettis Autobiografie

Dr. Shaimaa Ahmed Elsaghir Tawfik

مجلة وادي النيل للاراسات والبحوث الإنسانية والاجتماعية والتربوية (مجلة علمية محكمة)

Ich will sie [die Erinnerung] so intakt belassen, wie sie dem Menschen, der für seine Freiheit besteht, zugehört, und verhehle nicht meinen Abscheu vor denen, die sich herausnehmen, sie chirurgischen Eingriffen so lange auszusetzen, bis sie der Erinnerung aller übrigen gleicht. Mögen sie an Nasen, Lippen, Ohren, Haut und Haaren herumoperieren, soviel sie mögen, mögen sie ihnen, wenn es denn sein muss, andersfarbige Augen einsetzen, auch fremde Herzen, die ein Jährchen länger schlagen, mögen sie alles betasten, stutzen, glätten, gleichen, aber die Erinnerung sie sollen lassen stân.

Anhand der oben erwähnten Darstellung einiger Beispiele der Werke Canettis wird davon ausgegangen, dass das Thema literarische Erinnerung und autobiografisches Gedächtnis Gegenstand fast seines gesamten literarischen Schaffens ist. Dieses Thema soll nun exemplarisch am Roman Die gerettete Zunge kritisch vorgestellt werden.

\section{Die Konstruktion von Gedächtnis und Erinnerung in Canettis Die gerettete Zunge}

Im Alter von zweiundsiebzig Jahren veröffentlicht Canetti Die gerettete Zunge, den ersten Band seiner autobiografischen Trilogie. Diese Autobiografie schildert den Verlauf seines Lebens von 1905 bis 1921 und beschreibt die verschiedenen Länder, in denen er und seine Familie leben. Canettis Text wird oft als teleologischer Text gelesen, als strukturierte Darstellung der Entwicklung des Autors von Die Blendung und Masse und Macht.

Elias Canetti kündigt das autobiografische Gedächtnis ab der ersten Seite seines Romans an, sodass der anekdotische Stoff von Canettis Erinnerung inspiriert ist. Dabei steht das Wort Erinnerung in der ersten Zeile des Romanes, so äußert er: 
(ISSN : 2536 - 9555)

Meine frühste Erinnerung ist in Rot getaucht. Auf dem Arm eines Mädchens komme ich zu einer Tür heraus, der Boden vor mir ist rot, und zur Linken geht eine Treppe hinunter, den ebenso rot ist. Gegenüber von uns, in selber Höhe, öffnet sich eine Türe und ein lächelnder Mann tritt heraus, der freundlich auf mich zugeht. Er tritt ganz nahe an mich heran, bleibt stehen und sagt zu mir: Zeig die Zunge! Ich strecke die Zunge heraus, er greift in seine Tasche, zieht ein Taschenmesser hervor, öffnet es und führt die Klinge ganz nah an meine Zunge heran. Er sagte: »Jetzt schneiden wir ihm die Zunge ab.« Ich wage es nicht, die Zunge zurück zu ziehen, er kommt immer näher, gleich wird er sie mit der Klinge brühen. Im letzten Augenblick zieht er das Messer zurück, sagt: »Heute noch nicht, morgen.« Er klappt das Messer wieder zu und steckt es in seine Tasche. (Canetti 1985, S. 11)

Laut dem oben genannten Zitat aus der geretteten Zunge beginnt Canetti über die frühsten Erinnerungen an seine Kindheit in Rustschuk (Bulgarien), wo er 1905 geboren wurde (vgl. Barnouw 1979, S. 2). Diese Erinnerung, die er so lange in seinem autobiografischen Gedächtnis aufbewahrt hatte, präsentiert eine Szene der Gewalt, die sich gegen die Zunge richtet, die für die Sprache steht. Die Zunge ist ein wichtiges Leitmotiv im Rahmen der Autobiografie. Deshalb beschäftigen sich selbstverständlich viele Kritiker damit, eine genaue Analyse ihrer Symbolik darzustellen. Die Zunge steht hier für die Sprache, die spätere eine wichtige Rolle im Leben des Nobelpreisträgers gespielt hat. Die Eröffnungsszene, die mit ihrer kraftvollen Ikonografie die existenzielle Bedrohung der Kastration nachstellt, eine Bedrohung, die durch ihre Aufschiebung noch mächtiger ist, ist die erste in einer Reihe von Kindheitserinnerungen, die von sexueller Aggression und der Angst, verschlungen zu werden, handeln.

Die gerettete Zunge besteht aus fünf Teilen wie Die Fackel im Ohr und Das Augenspiel, wobei das Buch wie viele Autobiografien dem chronologischen Prinzip unterworfen ist. 


\section{Die literarische Konstruktion von Gedächtnis und Erinnerung in Elias \\ Canettis Autobiografie}

Dr. Shaimaa Ahmed Elsaghir Tawfik

مجلة وادي النيل للاراسات والبحوث الإنسانية والاجتماعية والتربوية (مجلة علمية محكمة)

Hierbei verwendet Canetti eine chronologische bzw. memorialistische Erzählweise, um die einheitliche Wertung der eignen biografischen Fakten zu bewahren. Canetti bemüht sich in der geretteten Zunge seiner Autobiografie um größere Genauigkeit und beschränkt sich darauf, die Funktion der Erinnerung und das autobiografische Gedächtnis anzudeuten. Diese Erinnerung funktioniert im Text sehr gut, wenn er Erlebnisse in der Vergangenheitsform darstellt. Dabei stellt diese Erinnerung laut Misch „den Grundbaustein des Lebens, jenes den Grundbaustein der Autobiographie dar" (Moser 2013, S. 6). So formuliert Canetti negativ seine Erinnerung an ein Spiel, dass sein Vater an Purim (ein jüdisches Fest) mit ihm gespielt hatte:

Eines Nachts, ich war schließlich doch eingeschlafen, weckte mich ein riesiger Wolf, der sich über mein Kinderbett neigte. Eine lange, rote Zunge hing ihm aus dem Mund und er fauchte fürchterlich. Ich schrie aus Leibeskräften: »Ein Wolf! Ein Wolf!« Niemand hörte mich, niemand kam, ich schrie immer gellender und weinte. Da kam eine Hand hervor, griff an die Ohren des Wolfs und zog seinen Kopf herunter. Dahinter stand der Vater und lachte. (Canetti 1977, S. 33)

Die gerettete Zunge weist wichtige Strukturmerkmale der Autobiografie auf, die die Rolle der Erinnerung und des Gedächtnisses andeuten. Hierbei zeichnet sich das Werk durch retrospektive Schilderung aus, wo Canetti aus der Perspektive rückblickender Erinnerung dem Leser seine Geschichte erzählt. Dadurch lässt er keinen Zweifel, dass der Erinnerung somit eine konstitutive Bedeutung für seine Autobiografie zukommt. Als ein weiteres Strukturmerkmal ist zu erwähnen, dass Die gerettete Zunge ein markantes Ereignis im Mittelpunkt der Handlung darstellt, das für Canettis Leben sowie seine literarischen Werke von großer Bedeutung ist, und zwar der frühe Tod des Vaters. Aus dieser Urszene entsteht ein canettischer Begriff - Tod-Feindschaft 
مجلة وادي النيل للاراسات والبحوث الإنسانية والاجتماعية والتربوية (مجلة علمية محكمة)

(ISSN : 2536 - 9555)

-, der in den meisten Werken Canettis auftaucht. Canettis Versuch, das Leben festzuhalten, die Wendung gegen den Tod können sicher als Grundmuster seines autobiografischen Schreibens vor allen anderen nachvollzogen werden.

Durch die beiden erwähnten Urszenen konnte Canetti die Authentizität der Erinnerung des Einzelnen im Zeitalter multipler und fragmentierter Identitäten bewahren. Der Anspruch auf Authentizität ist dadurch eines der wichtigsten Strukturmerkmale seiner Autobiografie.

\subsection{Erinnerung vs. Erzähler}

Wie zuvor erwähnt wurde, zeichnet sich die Autobiografie von Elias Canetti und insbesondere Die Gerettete Zunge durch retrospektive Schilderung, Gestaltung des Lebenszusammenhänge und die Betrachtung der Wechselwirkung zwischen Ich und Umwelt aus. Darüber hinaus ist die Ich-Form die verwendete Erzählform im Rahmen der Handlung. Zudem ist der Erzähler selbst der Autor, der in seiner Identität dem „Helden“ der Selbstbiografie entspricht. Es ist bemerkenswert, dass die dritte Person manchmal auftaucht, wo Canetti Distanz zwischen sich und die Erlebnisse bzw. Ereignisse bringen kann.

\subsubsection{Erinnerung und der Zeitpunkt des Erzählens}

Es gibt viele Bilder der Erinnerung in der Autobiografie Canettis, einschließlich des Bewusstseinsstroms und Monologs, Träume, Beschwörungen aus dem Gedächtnis, Mitteilungen, Aussagen und Berichte. So erfasste die horizontale Abfolge der zentralen Szenen im Roman nicht allein die Erzähltechniken, da der Autor Erzählräume schuf, um verschiedene Erinnerungsbilder zu verwenden, die dafür sorgten, dass die Komponenten der Erzählung in der Zeit verstreut wurden.

Um den Zeitpunkt des Erzählens irgendein literarisches

Werk zu identifizieren, müssen wir zunächst die Art der Beziehung 


\section{Die literarische Konstruktion von Gedächtnis und Erinnerung in Elias \\ Canettis Autobiografie}

Dr. Shaimaa Ahmed Elsaghir Tawfik

مجلة وادي النيل للاراسات والبحوث الإنسانية والاجتماعية والتريوية (مجلة عمية محكمة)

zwischen dem Zeitpunkt des Erzählens und dem Erzählten feststellen. Wenn wir Die gerettete Zunge genau lesen, erschließen wir, dass unser Autor das Präteritum als Tempus für sein Buch ausgewählt hat. Da das Phänomen Erinnerung fundamental auf dem Charakter des Vergangenen basiert, bleibt auch das Konstruieren der Erinnerung im Verhältnis zum Zeitpunkt des Erzählens selbstverständlich dieselbe zeitlose Vergangenheit. Der Roman beginnt mit einer Selbsterzählung des Tagebuchs des Erzählers mit seiner Familie in seiner Heimatstadt Rustschuk mit der Szene eines Mannes, der in der klaren und expliziten Präsenz des Romanciers im Text ihm die Zunge abschneiden will. Dabei kann der Leser Canetti im Mittelpunkt der Handlung als bewussten Narrator berücksichtigen, der die Schlüssel zu seinem Erzählspiel hat.

Elias Canetti hat aus zwei unterschiedlichen Erzählperspektiven die Geschichte dargestellt: Zum einen hat er sich in das vergangene Geschehen hineinversetzt, zum anderen erzählt er aus der Perspektive des auktorialen Erzählers, der dem Leser den Eindruck gibt, dass er heute seine Geschichte erzählt. Dabei erzählt er aus der Position des Wissens, da er alles über die handelnden Figuren in seiner Erzählung weiß.

\subsubsection{Erinnerungen und der Ort des Erzählens}

Der Ort im menschlichen Gedächtnis ist ein besonderer nostalgischer Zustand, dessen Kraft im Leben und in der Erinnerung nicht entzogen werden kann, und er wird umständlich und leidvoll, wenn es um das Schreiben geht. Vielleicht ist die canettische Erinnerung von Zärtlichkeit und psychologischer Bindung an die Geografie geprägt. Die Handlung des Buches bewegt sich an vielen Orten in der Erinnerung zwischen seinem Gedächtnis und der Erinnerung an die Familie, insbesondere seine Mutter, die ihr Leben zurückgewinnt und ihre Fäden sammelt, um sich in seiner Erzählung zu bilden. 
(ISSN : 2536 - 9555)

Wie zuvor bereits ausgeführt, hat Canetti den ersten Band seiner Autobiografie in fünf Episoden untergegliedert, in jeder von denen die Erinnerung Canettis an einen Ort von immenser Bedeutung ist. Dabei kann er festhalten, an welchem Punkt seines Lebens er bestimmte Erfahrungen und Begegnungen erlebte, denn alles steht in seinem Gedächtnis in einem dynamischen Zusammenhang zu einem bestimmten Ort des Erzählens. In Die gerettete Zunge sind dies die Städte Rustschuk, Manchester, Wien, Zürich-Scheuchzerstraße, Zürich-Tiefbrunnen, Frankfurt, Berlin, und Grinzin, wo Canetti zwischen 1905 bis 1921 lebte.

In Rustschuk lernte der junge Elias laut Barnouw heftige Gefühle kennen: Angst, Liebe und Hass. Seine Eltern verbanden Rustschuk mit erdrückender Unterdrückung und Tyrannei (vgl. Di Maio 2004, S. 175). 1911 siedelte die Familie Canetti nach Manchester um. Manchester verkörpert den Ort, wo er dem Tod zum ersten Mal begegnet ist. Hier in Manchester sollte der gesunde Vater so früh sterben. 1913 übersiedelte Canetti mit seiner Mutter nach Wien, wo sie die Zeit zwischen 1913 bis 1916 verbrachten. Dieser Ort steht in seinem Gedächtnis für die reale Begegnung mit der deutschen Sprache. Im Folgenden erinnert er sich an seine Mutter und wie er am Anfang die deutsche Sprache in Wien gelernt hat:

So zwang sie mich in kürzester Zeit zu einer Leistung, die über die Kräfte jedes Kindes ging, und dass es ihr gelang, hat die tiefere Natur meines Deutsch bestimmt, es war eine spät und unter wahrhaftigen Schmerzen eingepflanzte Muttersprache. Bei diesen Schmerzen war es nicht geblieben, gleich danach eine Periode des Glücks, und das hat mich unlösbar an diese Sprache verbunden. (G. Z., S. 100)

Die letzte Station in diesem Band ist Zürich, hier lebte Canetti ab 1916 bis 1921. Zürich war ein intellektuelles Paradies, ein Ort, an dem der junge Canetti ungeheure Wissensmengen aufnehmen konnte, deshalb konnte betrachtet werden, dass Canettis Erinnerungen an Zürich sehr glückliche sind. 
Die literarische Konstruktion von Gedächtnis und Erinnerung in Elias

Canettis Autobiografie

Dr. Shaimaa Ahmed Elsaghir Tawfik

مجلة وادي النيل للاراسات والبحوث الإنسانية والاجتماعية والتربوية (مجلة علمية محكمة)

\section{Fazit}

Die vorliegende Studie möchte am Beispiel von Canettis Die gerettete Zunge aufzeigen, wie die individuelle Erinnerung und das Gedächtnis in literarischen Autobiografien konstruiert werden und welche Konzepte des Gedächtnisses und der Erinnerung dabei entwickelt werden. Dabei wurde deutlich, dass Canettis Erinnerungsepisoden eine bisweilen große thematische Bandbreite beikommen und individuelle Erinnerungen in seiner gesamten literarischen Leistung in Erscheinung treten. Im Vordergrund hat er das autobiografische Erinnern und sein Ich immer wieder neu dargestellt, um es an die veränderten Lebensumstände anzupassen. Laut der vorliegenden Untersuchung lässt sich zusammenfassen, dass Elias Canetti als Autobiograf und Schriftsteller besonders geprägt von seinen Welterfahrungen, insbesondere von Ereignissen, Orten, Menschen, Gespräche und Lektüre ist. Somit gelang es ihm im Hinblick auf seine autobiografische Erinnerung zu dichten.

\section{Literaturverzeichnis}

- Barnouw, Dagmar. Elias Canetti. Stuttgart: Metzler, 1979.

- Canetti, Elias. "Die gerettete Zunge." Geschichte einer Jugend 29 (1977).

- Di Maio, Irene Stocksieker. "Space in Elias Canetti's Autobiographical Trilogy." A Companion to the Works of Elias Canetti (2004): 175.

- Erll, Astrid. Kollektives Gedächtnis und Erinnerungskulturen: Eine Einführung. Springer-Verlag, 2017.

- Gruber, Thomas. Gedächtnis. Springer-Verlag, 2018.

- Moser, Christian. Gedächtnis und Erinnerung in der Autobiographie. FernUniversität, 2013. 
(ISSN : 2536 - 9555)

- Schenk, Klaus: Erinnerendes Schreiben. Zur Autobiographik der 70er Jahren und ihren didaktischen Konsequenzen, in Klinger, Judith, and Gerhard Wolf, eds. Gedächtnis und kultureller Wandel: Erinnerndes Schreiben, Perspektiven und Kontroversen. Walter de Gruyter, 2009.

- Tulving, Endel: Episodic and Semantic Memory. In: Organisation of Memory. Hg.v.. E. Tulving und W. Donaldson. New York 1972, S. 381-402. In Moser, Christian. Gedächtnis und Erinnerung in der Autobiographie. FernUniversität, 2013.

- Wendy A. Suzuki, PhD: Associative learning and the hippocampus in Psychological Science Agenda February 2005, in:

https://www.apa.org/science/about/psa/2005/02/suzui. 